\title{
The Szemerédi-Petruska conjecture for a few small values
}

\author{
Adam S. Jobson ${ }^{1} \cdot$ André E. Kézdy ${ }^{1}$. Jenő Lehel ${ }^{1,2}$ (D)
}

Received: 29 September 2020 / Revised: 24 February 2021 / Accepted: 14 March 2021 /

Published online: 7 May 2021

(c) The Author(s) 2021

\begin{abstract}
Let $H$ be a 3-uniform hypergraph of order $n$ with clique number $\omega(H)=k$. Assume that the union of the $k$-cliques of $H$ equals its vertex set, the intersection of all maximum cliques of $H$ is empty, but the intersection of all but one $k$-clique is non-empty. For fixed $m=n-k$, Szemerédi and Petruska conjectured the sharp bound $n \leqslant\left(\begin{array}{c}m+2 \\ 2\end{array}\right)$. In this note the conjecture is verified for $m=2,3$ and 4 .
\end{abstract}

Keywords 3-Uniform hypergraph $\cdot$ Szemerédi-Petruska conjecture $\cdot \tau$-Critical hypergraph $\cdot$ Intersecting set pair systems $\cdot \tau$-Critical graph

Mathematics Subject Classification 05D05 · 05D15 · 05C65

\section{Introduction}

Let $H$ be a 3-uniform hypergraph of order $n$ with clique number $\omega(H)=k$. Assume that the union of the $k$-cliques of $H$ equals its vertex set, the intersection of its maximum cliques is empty, but the intersection of all but one maximum clique is non-empty. For fixed $m=n-k$ Szemerédi and Petruska [8] conjectured the tight bound $n \leqslant\left(\begin{array}{c}m+2 \\ 2\end{array}\right)$.

Here is the construction that shows this bound is sharp for infinitely many $k \geqslant 3$. For $m \geqslant 0$ let $k-1=\left(\begin{array}{c}m+1 \\ 2\end{array}\right)$, set $n=m+k$, and let $X, Y$ be disjoint sets such that $|X|=k-1,|Y|=m+1$. Let $X=\left\{x_{1}, \ldots, x_{k-1}\right\}$ and let $\left\{p_{1}, \ldots, p_{k-1}\right\}$ be the set of all 2-element sets of $Y$. For every $i=1, \ldots, k-1$ set $N_{i}=\left(X \backslash\left\{x_{i}\right\}\right) \cup p_{i}$. All triples in some $N_{i}, i=1, \ldots, k-1$, define a 3 -uniform hypergraph $H$ on the $n$-element

Jenő Lehel

lehelj@renyi.hu

Adam S. Jobson

adam.jobson@louisville.edu

André E. Kézdy

kezdy@louisville.edu

1 Department of Mathematics, University of Louisville, Louisville, KY 40292, USA

2 Alfréd Rényi Institute of Mathematics, Reáltanoda utca 13-15, 1053 Budapest, Hungary 
vertex set $X \cup Y$. It is straightforward to check that $\left\{N_{1}, \ldots, N_{k-1}\right\}$ is the family of all maximum cliques of $H$, in particular $\omega(H)=k$, furthermore, $\bigcap_{i=1}^{k-1} N_{i}=\varnothing$. Notice also that any $k-2$ maximum cliques have a common vertex in $X$; therefore, $H$ realizes an extremal hypergraph and shows the bound is sharp.

Indeed it had been conjectured this example is the unique extremal example for $m \geqslant 4$.

In Sect. 2 we prove the Szemerédi-Petruska conjecture for $m=2,3$, and 4 . The proof, based on $\tau$-critical graphs, makes it possible to characterize all extremal hypergraphs of order $n=\left(\begin{array}{c}m+2 \\ 2\end{array}\right)$ for $m \leqslant 4$. It is worth noting that there are two-two non-isomorphic extremal hypergraphs for $m=2$ and 3; meanwhile, the extreme is unique for $m=4$ and isomorphic to the Szemerédi-Petruska construction described above.

The transversal number of a hypergraph (or a graph), $\tau(H)$, is defined as the minimum number of vertices in a transversal set containing a vertex from every edge of $H$. A hypergraph (or graph) is $\tau$-critical if it has no isolated vertex and the removal of every edge decreases its transversal number.

The Szemerédi-Petruska conjecture is equivalent with the statement that $\left(\begin{array}{c}m+2 \\ 2\end{array}\right)$ is the maximum order of a 3-uniform $\tau$-critical hypergraph with transversal number $m$, see [4] or [9, Problem 18 (a)]. For the maximum order, Tuza ${ }^{1}$ obtained the best known bound $\frac{3}{4} m^{2}+m+1$ using the machinery of $\tau$-critical hypergraphs.

An alternative approach to solve the conjecture is proposed by Jobson et al. [6], combining a decomposition process introduced by Szemerédi and Petruska [8] with the skew version of Bollobás's theorem [1], and using tools from linear algebra.

It turns out that the Szemerédi-Petruska conjecture has applications in extremal problems concerning convex sets in the plane, see Jobson et al. [5] and [7]. The validity of the Szemerédi-Petruska conjecture for small values carries relevant information pertaining to those combinatorial geometry problems.

\section{Proof of the conjecture for $m=2,3$, and 4}

In the proof presented here $\tau$-critical graphs play a key role. In particular, we use the tight bounds (1) and (2) shown below due to Erdős and Gallai [2] and Gyárfás and Lehel [3], respectively.

Lemma 2.1 ([2,3]) If $G=(V, E)$ is a $\tau$-critical graph with $\tau(G)=t$, then

$$
\begin{gathered}
|V| \leqslant 2 t \\
|V|+|E| \leqslant\left(\begin{array}{c}
t+2 \\
2
\end{array}\right) .
\end{gathered}
$$

Proposition 2.2 Let $m=2,3$, or 4, and $n>m$. If $H$ is a 3-uniform hypergraph of order $n$ with clique number $\omega(H)=n-m=k \geqslant 3$, and the intersection of the $k$-cliques of $H$ is empty, then $n \leqslant\left(\begin{array}{c}m+2 \\ 2\end{array}\right)$.

\footnotetext{
${ }^{1}$ Personal communication.
} 
Proof Let $\mathcal{N}=\left\{N_{1}, \ldots, N_{\ell}\right\}$ be a collection of $k$-cliques of $H=(V, E)$ such that $\bigcap_{i=1}^{\ell} N_{i}=\varnothing$, but $\bigcap_{j \neq i} N_{j} \neq \varnothing$ for all $i=1, \ldots, \ell$. Notice that if $\bigcup N_{i} \neq V$, say $v \in V \backslash \bigcup N_{i}$, then we may consider $H-v$ thereby reducing both $n$ and $m$ by one. Hence we may assume $\bigcup_{i=1}^{\ell} N_{i}=V$ and $|V|=n$. Furthermore, we assume that $\ell \geqslant 3$, since $H$ needs to have at least two cliques, and the claim is obvious for $\ell=2$, even if $H$ consists of two disjoint $k$-cliques.

Szemerédi and Petruska [8, Lemma 4] observed that each $N_{i}, i=1, \ldots, \ell$, contains a private pair: $p_{i} \subset V,\left|p_{i}\right|=2$, such that $p_{i} \subset N_{j}$ if and only if $i=j$. Let $G=(V, E)$ be the graph of the private pairs $p_{i}=\left\{a_{i}, b_{i}\right\}, 1 \leqslant i \leqslant \ell$, as its edges.

Set $M_{i}=V \backslash N_{i}$, and notice that $\left|M_{i}\right|=n-k=m$. By the definition of private pairs, $\left(p_{i}, M_{i}\right), 1 \leqslant i \leqslant \ell$, form an intersecting $(2, m)$-system: $p_{i} \cap M_{j}=\varnothing$ if and only if $i=j$. Furthermore, $\bigcup p_{i} \subseteq \bigcup M_{i}=V\left(\right.$ since $\left.\bigcap N_{i}=\varnothing\right)$.

For an edge $p_{j}=\left\{a_{j}, b_{j}\right\}$ of $G$, define the truncated subgraph $G \backslash p_{j}$ on vertex set $V \backslash\left\{a_{j}, b_{j}\right\}$ by including $p_{h} \backslash\left\{a_{j}, b_{j}\right\}$ as an edge or a loop, for all $h \neq j$. Since $M_{j} \cap p_{h}=M_{j} \cap\left(p_{h} \backslash p_{j}\right) \neq \varnothing$ for all $h \neq j$, we have

$$
\tau\left(G \backslash p_{j}\right) \leqslant\left|M_{j}\right|=m,
$$

for every $1 \leqslant j \leqslant \ell$. For each edge $p_{j} \in G$ we define the weight

$$
w\left(p_{j}\right)=m-\tau\left(G \backslash p_{j}\right) .
$$

Let $G-p_{j}$ denote the graph obtained by the removal of edge $p_{j}$ from $G$ (and nothing else). Obviously we have

$$
\tau(G)-1 \leqslant \tau\left(G-p_{j}\right) \leqslant \tau\left(G \backslash p_{j}\right) \leqslant m .
$$

The conditions $\bigcap N_{i}=\varnothing$ and (4) imply $2 \leqslant \tau(G) \leqslant m+1$. Furthermore, $0 \leqslant w\left(p_{j}\right)=$ $m-\tau\left(G \backslash p_{j}\right) \leqslant m-\tau(G)+1$.

Let $V_{0}(G)=\bigcup_{p \in E} p$, set $Z(G)=V \backslash V_{0}(G)$ and $w(G)=\sum_{p \in E} w(p)$.

Lemma $2.3|V|=\left|V_{0}(G)\right|+|Z(G)| \leqslant\left|V_{0}(G)\right|+w(G)$.

Proof Set $V_{0}=V_{0}(G)$, and $Z_{j}=M_{j} \backslash V_{0}$. Observe that (3) implies $\left|M_{j} \cap V_{0}\right| \geqslant \tau\left(G \backslash p_{j}\right)$, hence $\left|Z_{j}\right|=\left(\left|M_{j}\right|-\left|M_{j} \cap V_{0}\right|\right) \leqslant m-\tau\left(G \backslash p_{j}\right)=w\left(p_{j}\right)$. In words, $M_{j}$ has at most $w\left(p_{j}\right)$ vertices not in $V_{0}$. Thus $|Z(G)| \leqslant \sum_{j=1}^{\ell}\left|Z_{j}\right| \leqslant \sum_{j=1}^{\ell} w\left(p_{j}\right)=w(G)$, and the claim follows.

Now we break the proof of Proposition 2.2 into three cases.

Case 1: $\tau(G)=2$. Let $\{u, v\}$ be a transversal set of $G$, set $V_{0}=V_{0}(G)$, and let $X=\left\{a \in V_{0} \backslash\{v\}: u a \in E\right\}$ and $Y=\left\{a \in V_{0} \backslash\{u\}: v a \in E\right\}$. Set $x=|X|, y=|Y|$, and $z=|X \cap Y|$.

Case 1.1: $Y \subseteq X$. In this case $\left|V_{0}\right|=|X \cup\{u, v\}|=x+2$. If $q=\{u, v\} \in E$, then $\tau(G)=2$ implies $\tau(G \backslash q)=|X|$, hence $w(q)=m-\tau(G \backslash q)=m-x$; if $q \notin E$, 
define $w(q)=0$. We also have

$$
\begin{aligned}
& \tau(G \backslash u a)=|X \backslash\{a\}|=x-1, \quad w(u a)=m-x+1 \text { if } a \in X \backslash Y ; \\
& \tau(G \backslash u a)=|(X \backslash\{a\}) \cup\{v\}|=x, \quad w(u a)=m-x \text { if } a \in Y ; \\
& \tau(G \backslash v a)=|(Y \backslash\{a\}) \cup\{u\}|=y, \quad w(v a)=m-y \text { if } a \in Y .
\end{aligned}
$$

Thus the bound in Lemma 2.3 becomes

$$
\begin{aligned}
|V| & \leqslant\left|V_{0}\right|+w(G)=(x+2)+w(q)+\sum_{p \neq q} w(p) \\
& \leqslant(x+2)+(m-x)+(x-y)(m-x+1)+y(m-x)+y(m-y) \\
& =m+2+x(m-x+1)+y(m-y-1) \\
& \leqslant m+2+\left\lfloor\frac{m+1}{2}\right\rfloor \cdot\left\lceil\frac{m+1}{2}\right\rfloor+\left\lfloor\frac{m-1}{2}\right\rfloor \cdot\left\lceil\frac{m-1}{2}\right\rceil \leqslant\left(\begin{array}{c}
m+2 \\
2
\end{array}\right) .
\end{aligned}
$$

Case 1.2: $X \backslash Y \neq \varnothing$ and $Y \backslash X \neq \varnothing$. In this case $\left|V_{0}\right|=x+y-z+2$, and if $q=u v \in E$, then $\tau(G \backslash q)=x+y-z, w(q)=m-x-y+z$; furthermore, $\tau(G \backslash u a)=x, w(u a)=m-x$ if $a \in X$, and $\tau(G \backslash v a)=y, w(v a)=m-y$ if $a \in Y$. Thus by applying Lemma 2.3 we obtain the bound

$$
\begin{aligned}
|V| & \leqslant(x+y-z+2)+(m-x-y+z)+x(m-x)+y(m-y) \\
& \leqslant m+2+2\left\lfloor\frac{m}{2}\right\rfloor \cdot\left\lceil\frac{m}{2}\right\rceil \leqslant\left(\begin{array}{c}
m+2 \\
2
\end{array}\right) .
\end{aligned}
$$

Therefore, if $\tau(G)=2$ then $n \leqslant\left(\begin{array}{c}m+2 \\ 2\end{array}\right)$ follows for every $m \geqslant 2$.

Case 2: $\tau(G)=m+1$ or $m$. Assume $\tau(G)=m+1$. By (4), $0 \leqslant w\left(p_{i}\right)=m-$ $\tau\left(G \backslash p_{i}\right) \leqslant m-\tau\left(G-p_{i}\right)$, for every $1 \leqslant i \leqslant \ell$, thus we have $\tau\left(G-p_{i}\right) \leqslant m$. Therefore, $G$ is a $\tau$-critical graph and the bound (1) in Lemma 2.1 yields $|V| \leqslant 2(m+1)$. For $m \geqslant 2,|V| \leqslant 2(m+1) \leqslant\left(\begin{array}{c}m+2 \\ 2\end{array}\right)$ follows.

Assume now that $\tau(G)=m$. Notice that $w\left(p_{i}\right)=0$ means $M_{i} \subset V_{0}(G)$. By successively removing edges from $G$ with $w\left(p_{i}\right)=0$ we conclude with a subgraph $G^{*}$ such that $\tau\left(G^{*}\right)=m$ and $0<w(p)=m-\tau\left(G^{*} \backslash p\right) \leqslant m-\tau\left(G^{*}-p\right)$, for every $p \in E^{*}$. Hence $G^{*}$ is a $\tau$-critical graph. We claim that $|V| \leqslant\left|V^{*}\right|+\left|E^{*}\right|$, and from the bound (2) in Lemma 2.1 we obtain $|V| \leqslant\left|V^{*}\right|+\left|E^{*}\right| \leqslant\left(\begin{array}{c}m+2 \\ 2\end{array}\right)$.

The claim $|V| \leqslant\left|V^{*}\right|+\left|E^{*}\right|$ follows by repeatedly applying the next general $^{2}$ lemma.

Lemma 2.4 If $\ell \geqslant 3$ and $w\left(p_{\ell}\right)=0$, then $G^{\prime}=G-p_{\ell}$ satisfies

$$
\left|V_{0}(G)\right|+w(G) \leqslant\left|V_{0}\left(G^{\prime}\right)\right|+w\left(G^{\prime}\right) .
$$

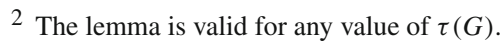


Proof For $1 \leqslant i \leqslant \ell-1$, define $w^{\prime}\left(p_{i}\right)=m-\tau\left(G^{\prime} \backslash p_{i}\right)$. Since a transversal set in $G \backslash p_{i}$ is also a transversal set in $G^{\prime} \backslash p_{i}$, we have $\tau\left(G \backslash p_{i}\right) \geqslant \tau\left(G^{\prime} \backslash p_{i}\right)$. Then we obtain

$$
w\left(G^{\prime}\right)=\sum_{j=1}^{\ell-1} w^{\prime}\left(p_{j}\right)=\sum_{j=1}^{\ell-1}\left(m-\tau\left(G^{\prime} \backslash p_{i}\right)\right) \geqslant \sum_{j=1}^{\ell-1}\left(m-\tau\left(G \backslash p_{i}\right)\right)=w(G) .
$$

If $p_{\ell}$ is neither an isolated edge nor a pendant edge, then $V_{0}\left(G^{\prime}\right)=V_{0}(G)$, therefore $\left|V_{0}(G)\right|+w(G) \leqslant\left|V_{0}\left(G^{\prime}\right)\right|+w\left(G^{\prime}\right)$ follows.

If $p_{\ell}$ is an isolated edge in $G$, then $\left|V_{0}\left(G^{\prime}\right)\right|=\left|V_{0}(G)\right|-2$. Because $w\left(p_{\ell}\right)=0$, we have $\tau(G)=\tau\left(G \backslash p_{\ell}\right)+1=m-w\left(p_{\ell}\right)+1=m+1$. Moreover, $G$ is $\tau$-critical, since by (4),

$$
\tau(G)-1 \leqslant \tau\left(G-p_{j}\right) \leqslant m=\tau(G)-1,
$$

for every $1 \leqslant j \leqslant \ell$. Then $w\left(p_{j}\right)=m-\tau\left(G \backslash p_{j}\right)=0$ thus $w(G)=0$. Observe that $G^{\prime}$ is $\tau$-critical, as well, with $\tau\left(G^{\prime}\right)=\tau(G)-1=m$, therefore, $w^{\prime}\left(p_{j}\right)=$ $m-\tau\left(G^{\prime} \backslash p_{j}\right)=m-\tau\left(G^{\prime}-p_{j}\right)=1$. Then by the definition of the edge weights, we have $w\left(G^{\prime}\right)=\sum_{j=1}^{\ell-1} 1=\left|E^{\prime}\right|$. Since $\left|E^{\prime}\right| \geqslant m \geqslant 2$, we obtain $\left|V_{0}(G)\right|+w(G)$ $=\left|V_{0}(G)\right| \leqslant\left(\left|V_{0}(G)\right|-2\right)+\left|E^{\prime}\right|=\left|V_{0}\left(G^{\prime}\right)\right|+w\left(G^{\prime}\right)$.

If $p_{\ell}$ is a pendant edge, then $\left|V_{0}\left(G^{\prime}\right)\right|=\left|V_{0}(G)\right|-1$. For any $p_{i} \cap p_{\ell} \neq \varnothing$, $\tau\left(G^{\prime} \backslash p_{i}\right)=\tau\left(G \backslash p_{i}\right)-1$, because a minimum transversal in $G \backslash p_{i}$ must use a vertex just for $p_{\ell}$, and this vertex is not required in a minimum transversal of $G^{\prime} \backslash p_{i}$. Hence $w^{\prime}\left(p_{i}\right)=w\left(p_{i}\right)+1$, which implies $w\left(G^{\prime}\right) \geqslant w(G)+1$, and $\left|V_{0}(G)\right|+w(G)=$ $\left(\left|V_{0}(G)\right|-1\right)+(w(G)+1) \leqslant\left|V_{0}\left(G^{\prime}\right)\right|+w\left(G^{\prime}\right)$ follows.

Because $2 \leqslant \tau(G) \leqslant m+1$, Steps 1 and 2 imply $n \leqslant\left(\begin{array}{c}m+2 \\ 2\end{array}\right)$, for $m=2,3$.

Case 3: $m=4, \tau(G)=3$. By Lemma 2.4 we may assume that all edges of $G$ have positive weights. Since $\tau(G)=3, G$ contains one of the four $\tau$-critical subgraphs in Fig. 1.

Case 3.1: $G$ contains a $K_{4}$. If $G$ had two more non-isolated vertices, $a$ and $b$, then $a b \notin E$, since $\tau(G)=3$. One edge from each of $a$ and $b$ to $K_{4}$ would result in an edge of zero weight. Hence $\left|V_{0}\right|=5$ or 4 , and $G$ can be obtained starting with a $K_{5}$, and successively removing edges incident with a common vertex, say $a$. The corresponding configurations are depicted in Fig. 2 (unlabeled edges have weight 1).

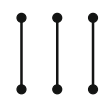

$3 K_{2}$

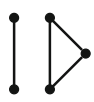

$K_{2}+C_{3}$

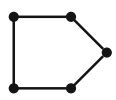

$C_{5}$

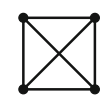

$K_{4}$

Fig. $1 \tau$-critical graphs $G$ with $\tau(G)=3$ 


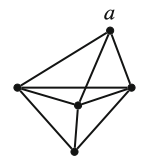

$n \leqslant 14$

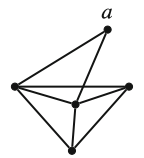

$n \leqslant 13$

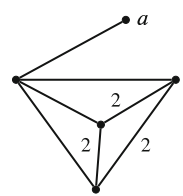

$n \leqslant 15$

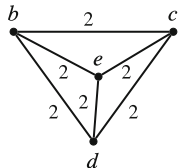

$n \leqslant 16$

Fig. $2 G$ has a $K_{4}$

The rightmost candidate in Fig. 2 can be rejected as follows. If $n=16>\left(\begin{array}{c}4+2 \\ 2\end{array}\right)$, then $Z_{i}=M_{i} \backslash V_{0}, 1 \leqslant i \leqslant 6$, are pairwise disjoint 2-element sets. Let $V_{0}=\{b, c, d, e\}$ and $Z_{i}=\left\{i, i^{\prime}\right\}$. The corresponding $(2, m)$-system is uniquely determined as follows: $p_{1}=b c, p_{2}=b d, p_{3}=b e, p_{4}=c d, p_{5}=c e, p_{6}=d e$, and $M_{1}=\left\{d, e, 1,1^{\prime}\right\}$, $M_{2}=\left\{c, e, 2,2^{\prime}\right\}, M_{3}=\left\{c, d, 3,3^{\prime}\right\}, M_{4}=\left\{b, e, 4,4^{\prime}\right\}, M_{5}=\left\{b, d, 5,5^{\prime}\right\}, M_{6}=$ $\left\{b, c, 6,6^{\prime}\right\}$.

We eliminate the 'fake' candidate by applying a general observation, the Triples test, as follows. If a set $f \subset V,|f|=3$, is such that $f \cap M_{i}=\varnothing$ for some $1 \leqslant i \leqslant \ell$, then $f \subset N_{i}$ and since $N_{i}$ is a clique of $H$, we have $f \in E$.

Let $N=V \backslash\{c, d, e\} ;|N|=n-3=n-(m-1)=k+1$. Notice that the four sets $M_{i} \backslash\{c, d, e\}, i=1,2,3,4$, are pairwise disjoint, thus every 3-element set $f \subset N$ is disjoint from some $M_{i}, 1 \leqslant i \leqslant 6$. Hence, by the Triples test, $f \in E$ for every $f \subset N$, $|f|=3$. Therefore, $N$ induces a clique of order $k+1$, a contradiction.

Case 3.2: $G$ has a 5-cycle and no $K_{4}$. Since $\tau(G)=3$, all edges of $G$ are incident with the 5-cycle. Figure 3 lists all configurations with no zero edge weights (unlabeled edges have weights 1$)$. All candidates have at most $\left(\begin{array}{c}4+2 \\ 2\end{array}\right)=15$ vertices.
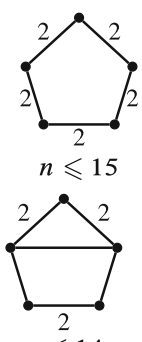

$n \leqslant 14$

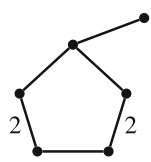

$n \leqslant 14$

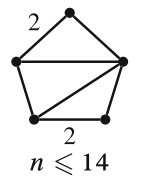

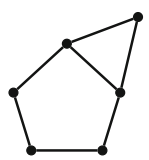

$n \leqslant 13$

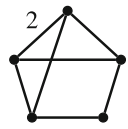

$n \leqslant 13$

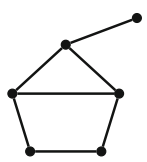

$n \leqslant 13$

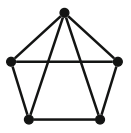

$n \leqslant 13$

Fig. $3 G$ has a 5-cycle and no $K_{4}$ 


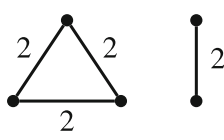

$n \leqslant 13$

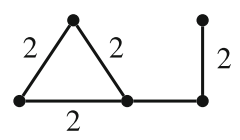

$n \leqslant 14$

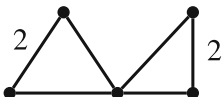

$n \leqslant 13$

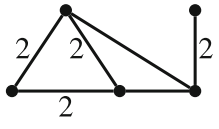

$n \leqslant 15$

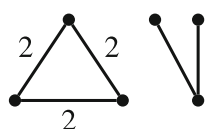

$n \leqslant 14$

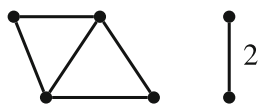

$n \leqslant 13$

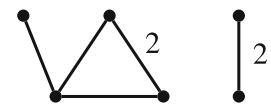

$n \leqslant 13$

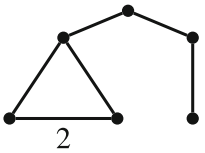

$n \leqslant 13$

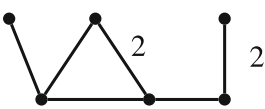

$n \leqslant 14$

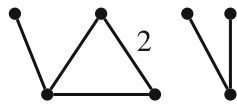

$n \leqslant 14$

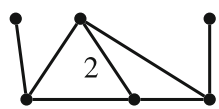

$n \leqslant 14$

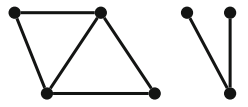

$n \leqslant 14$

Fig. $4 G$ has a triangle and no 5-cycle or $K_{4}$

Case 3.3: $G$ has a triangle, it has no 5-cycle and no $K_{4}$. The $\tau$-critical subgraph in $G$ is $K_{2}+K_{3}$. To get all candidates at most three edges and one new vertex can be added to $K_{2}+K_{3}$ by keeping the transversal number 3 and creating neither a 5-cycle nor a $K_{4}$. These graphs are listed in Fig. 4; all have at most 15 vertices.

Case 3.4: $G$ has no cycle. The $\tau$-critical subgraph in $G$ is $3 K_{2}$. To get all candidates from this one at most three edges can be added by keeping the transversal number 3 and creating no cycle. These graphs are listed in Fig. 5; all have at most 15 vertices.

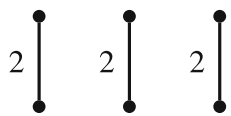

$n \leqslant 12$

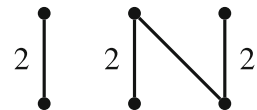

$n \leqslant 13$

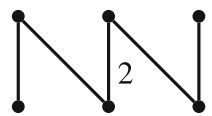

$n \leqslant 12$

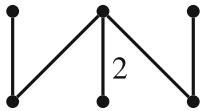

$n \leqslant 12$

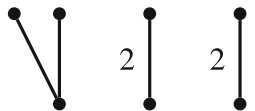

$n \leqslant 13$
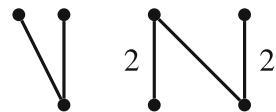

$n \leqslant 14$

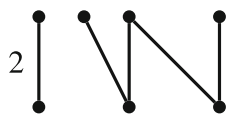

$n \leqslant 13$

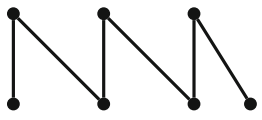

$n \leqslant 13$
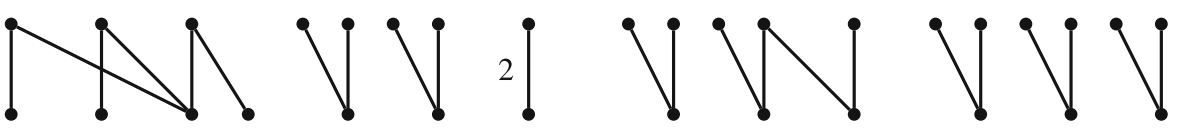

$n \leqslant 13$

$n \leqslant 14$

$n \leqslant 14$

$n \leqslant 15$ 
Funding Open access funding provided by ELKH Alfréd Rényi Institute of Mathematics.

Open Access This article is licensed under a Creative Commons Attribution 4.0 International License, which permits use, sharing, adaptation, distribution and reproduction in any medium or format, as long as you give appropriate credit to the original author(s) and the source, provide a link to the Creative Commons licence, and indicate if changes were made. The images or other third party material in this article are included in the article's Creative Commons licence, unless indicated otherwise in a credit line to the material. If material is not included in the article's Creative Commons licence and your intended use is not permitted by statutory regulation or exceeds the permitted use, you will need to obtain permission directly from the copyright holder. To view a copy of this licence, visit http://creativecommons.org/licenses/by/4.0/.

\section{References}

1. Bollobás, B.: On generalized graphs. Acta Math. Acad. Sci. Hungar. 16, 447-452 (1965)

2. Erdős, P., Gallai, T.: On the maximal number of vertices representing the edges of a graph. Közl. MTA Mat. Kutató Int. Budapest 6, 181-203 (1961)

3. Gyárfás, A., Lehel, J.: Order plus size of $\tau$-critical graphs. J. Graph Theory 96(1), 85-86 (2020)

4. Gyárfás, A., Lehel, J., Tuza, Zs.: Upper bound on the order of $\tau$-critical hypergraphs. J. Combin. Theory Ser. B 33(2), 161-165 (1982)

5. Jobson, A., Kézdy, A., Lehel, J.: Eckhoff's problem on planar convex sets (2019) (note)

6. Jobson, A.S., Kézdy, A.E., Pervenecki, T.: On a conjecture of Szemerédi and Petruska (2019). arXiv: $1904.04921 \mathrm{v} 2$

7. Jobson, A.S., Kézdy, A.E., Lehel, J., Pervenecki, T.J., Tóth, G.: Petruska's question on planar convex sets. Discrete Math. 343(9), \# 111956 (2020)

8. Szemerédi, E., Petruska, G.: On a combinatorial problem I. Studia Sci. Math. Hungar. 7, 363-374 (1972)

9. Tuza, Zs.: Critical hypergraphs and intersecting set-pair systems. J. Combin. Theory Ser. B 39(2), 134-145 (1985)

Publisher's Note Springer Nature remains neutral with regard to jurisdictional claims in published maps and institutional affiliations. 